\title{
JOSÉ SOTERAS MAURI Y LORENZO GARCÍA-BARBÓN. 3 (ESPACIOS) A CUBIERTO
}

\author{
Ignacio López Alonso
}

José Soteras y Lorenzo García-Barbón, coautores del Camp Nou junto a Francisco Mitjans, construyeron en la década de los cincuenta una serie de proyectos de uso público para actos religiosos y deportivos. Paradigmático del trabajo de Soteras fue el Altar Mayor del XXXV Congreso Eucarístico Internacional de Barcelona (1952), y ya en colaboración, el Palacio Municipal de Deportes de Barcelona (1954-1955) y el destruido Palacio de Deportes de Madrid (1955-1960). Es posible reseguir en estas obras una secuencia evolutiva, función de la relación entre forma y geometría de la estática; transformando la obligada austeridad económica del momento en virtud espacial.

Palabras clave: Barcelona, José Soteras, Lorenzo García-Barbón, Palacio de Deportes, X Congreso Eucarístico, Camp Nou, estructura, forma Keywords: Barcelona, José Soteras, Lorenzo García-Barbón, Palacio de Deportes, X Congreso Eucarístico, Camp Nou, structure, form

\section{EN TORNO A JOSÉ SOTERAS MAURI}

La figura de J osé Soteras M auri, hoy prácticamente relegada al olvido, ocupó en el primer periodo del franquismo el centro de la escena arquitectónica barcelonesa. De él apenas nos quedan referencias implantadas en la cultura popular arquitectónica de la ciudad, como la clásica frase "si quiere usted construir en las aceras, Ilame a Soteras". De al gún modo el papel de Soteras como arquitecto municipal vinculado al Régimen Franquista ha velado la percepción de la práctica profesional del Soteras arquitecto.

Esta desaparición en la literatura posterior de la arquitectura barcelonesa podría derivarse de esa íntima vinculación con las élites franco-falangistas de la primera etapa de la dictadura. Y, por qué no decirlo, de su posible enfrentamiento con aquellos que reescribieron la historia del momento. Difícilmente se podría calificar a J osé Soteras M auri de personaje no incómodo para la crítica estructurada de la arquitectura de la (Escuela de) Barcelona del tardofranquismo, empeñada en la reconstrucción postmoderna de la biografía de la futura élite arquitectónica de la ciudad.

En cualquier caso, se pueden aportar una serie de datos objetivos en torno a la figura de Soteras. Nacido en 1907, arquitecto desde 1930, fue socio del GATCPAC durante la Segunda República'. Entró en Barcelona en 1939 como teniente provisional del ejército golpista comandado por Francisco Franco ${ }^{2}$. Fue arquitecto municipal y posteriormente, director de la Oficina de Estudios de la Comisión Técnica de U rbanismo del Ayuntamiento de Barcelona, vocal de la Ponencia técnica de la Comisión Superior de Ordenación Provincial y jefe del Servicio de Extensión y Reforma del Ayuntamiento barcelonés 3 .

Como tal, inspiró el Plan Comarcal de B arcelona de 1953, conocido con el sobrenombre de "plan Soteras", así como diversos planeamientos puntuales en la ciudad de Barcelona. Cabe destacar entre ellos el "Plan de U rbanización de la Avenida del Generalísimo" (la actual Avenida Diagonal) o el "Proyecto de nuevas alineaciones y rasantes en la zona comprendida por las calles de Felipe II, Concepción A renal y Riera de Horta para el emplazamiento de viviendas del Congreso"4.

En paral elo, y casi siempre en colaboración con diferentes arquitectos e ingenieros, realizó una extensa obra arquitectónica, buena parte de ella a todas luces reseñable. U na producción identificada con una serie de valores comunes, propios de un racionalismo pragmático donde la estructura se convierte en elemento protagonista del proyecto.

Se podría calificar como clave en el proceso de formación de Soteras la figura del ingeniero I talo L auro. Conjuntamente desarrollaron desde los primeros cuarenta el proyecto para
1. PIZZA, Antonio; ROVIRA, Josep Ma, G.A.T.C.P.A.C. Una nueva arquitectura para una nueva ciudad. 1928-1939, COAC, Barcelona, 2006

2. Según consta en La Vanguardia Española, los tenientes provisionales de Ingenieros señores Soteras y Solá-Morales fueron los colaboradores técnicos de la comisión formada para la construcción de la Residencia de Oficiales de la Avenida Diagonal (en aquel momento, Avenida del Generalísimo Franco). "Labor concreta de reconstrucción militar y civil de Barcelona en los dos primeros años de su Era de liberación", La Vanguardia Española, 26 de enero de1941, p. 1.

3. "Crónica de la jornada. Urbanismo y urbanistas", La Vanguardia Española, 18 de noviembre de1954, p. 13.

4. RODRÍGUEZ, Carmen, "Les VCE: un projecte experimental amb aspiració modélica", A.A.V.V., Les Vivendes del Congrés Eucarístic de Barcelona. 1952-1962, Iniciativa Digital Politècnica, Barcelona, 2011, p. 16 
la fábrica Olivetti junto a la plaza de las G lorias. Este proyecto incorporaba las innovaciones humanistas derivadas de la política de la firma, ya aplicadas por Italo L auro en las oficinas de Ivrea, derivando en un banco de pruebas para proyectos posteriores. No en vano, B rufau y O biols califican a I talo L auro de "proyectista excelente y calculista riguroso" ${ }^{5}$. El proyecto de Olivetti fue utilizado en la Exposición del día M undial del U rbanismo de 1950 como imagen paradigmática de la industrialización del M ovimiento ${ }^{6}$. Dicha exhibición fue realizada en el Saló del Tinell de Barcelona en 1950 y contó con la asistencia del mismo Francisco Franco. En una muestra más de la oficialización de la arquitectura moderna en el Régimen tras la V A samblea Nacional de A rquitectos de 1949.

\section{CRITERIOS DE SELECCIÓN}

A fin de trazar una posible secuencia evolutiva en parte de la obra de J osé Soteras M auri, se han seleccionado tres proyectos, dos de ellos en colaboración con Lorenzo GarcíaBarbón, aún cuando por la extensión de su obra esta muestra podría ser mucho mayor. A fin de ser claro en su desarrollo, se ha enfocado el análisis en obra de pública concurrencia, y relacionada con actos de masas, cumpliendo con dos condiciones principales.

En primer lugar, se trata de tres casos donde la estructura tiene un valor preeminente en la configuración del espacio, bien sea interior o exterior. Con independencia de la escala del trabajo, todos ellos permiten una lectura del proyecto donde la estructura formal del conjunto se vincula intencionadamente con la legibilidad de las cuestiones propias de la estática.

En segundo lugar, estos casos permiten trazar una cierta reflexión o investigación respecto a ciertas constantes en esta obra conjunta. Podrían ayudar, por tanto, a detectar la continuidad de determinadas soluciones estructurales y sus modificaciones a través del análisis de la forma y el programa. Habilitarían, en consecuencia, la posibilidad de generar hipótesis en torno a un cierto progreso personal del tipo.

Para describir este proceso las obras seleccionadas se emplazan cronológicamente en un periodo relativamente breve de veinte años, entre 1952 y 1960. Un momento de amplios cambios en la escena sociopolítica local; una vez finalizada la autarquía. En el tránsito entre el nacional-catolicismo y el inicio de la ayuda americana en los primeros 50, "la primavera falangista" de 1957; y concluyendo con el Plan de Estabilización de 1959 y el aperturismo de la primera década de los 60.

La trayectoria descrita mediante los tres casos de análisis no es indiferente a la existencia de obras realizadas en paral elo donde se estudiaron elementos parciales aplicados posteriormente. Inevitablemente éstas se cruzarán en la línea descrita aportando datos o soluciones puntuales complementarias.

El A Itar M ayor del XXXV Congreso Eucarístico Internacional de Barcelona (1952) representa un punto de inflexión en la obra de Soteras, iniciándose una vinculación consecutiva entre la forma y la lógica de su solución estructural. U na evolución confirmada por el Palacio M unicipal de Deportes de Barcelona (1954-1955). Cuya solución se maduraría a posteriori en el destruido Palacio de Deportes de M adrid (1955-1960), donde se alcanzaría un punto de equilibrio entre estructura y forma.

\section{CONTRAPESOS}

El primero de los tres proyectos analizados es el A Itar M ayor del XXXV Congreso Eucarístico Internacional, instalación temporal realizada en Barcelona entre mayo y junio de 1952. Este se emplazó en la plaza Pio XII, sobre la entonces Avenida del Generalísimo. Su instalación sobre la actual Avenida Diagonal de Barcelona, en aquel momento apenas un trazado urbano sin asfaltar, debía responder simultáneamente a las necesidades simbólicas, de accesibilidad y de visibilidad.

El proyecto consistía en la erección de una estructura efímera para la protección del A Itar M ayor, centro del ritual litúrgico y uno de los escenarios de los actos de masas realizados en
5. BRUFAU, Robert; OBIOL, Agustín, "El diseño estructural en Barcelona durante el periodo 1950-1963", A.A.V.V. , en La arquitectura de los años cincuenta en Barcelona, ETSA Vallés-Direcció General d'Arquitectura i Habitatge de la Generalitat, Barcelona, 1987, p. 220

6. SOTERAS MAURI, José, "El Plan de Ordenación de Barcelona y su zona de influencia en la Exposición del Día de Urbanismo", en Cuadernos de Arquitectura, 1950, 14, p. 2. 


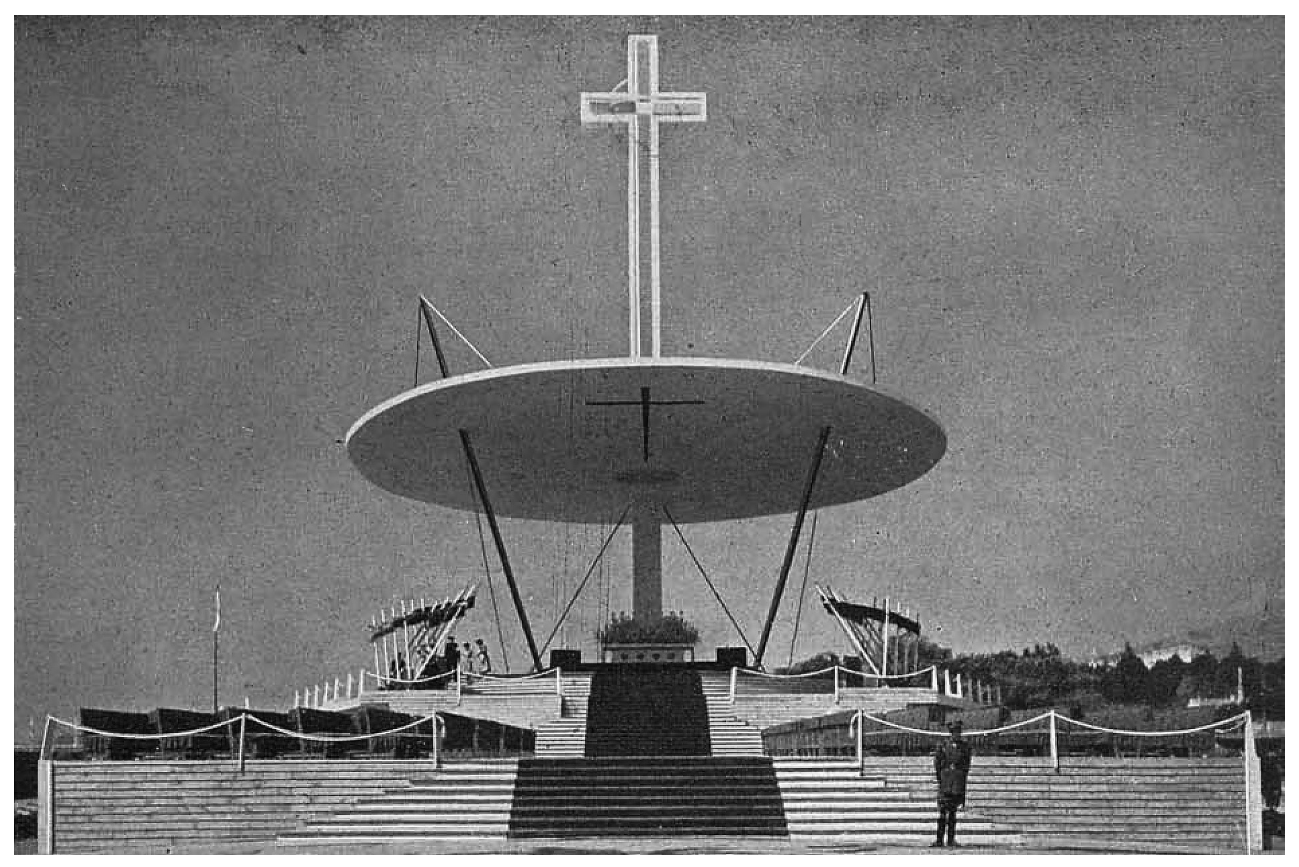

1

el congreso. Este elemento debía incorporar, además del espacio central del ara, una tribuna escal onada para disponer a prelatura y autoridades a cubierto.

El conjunto se caracterizó mediante una gran cruz de 35 metros de altura y un baldaquino circular de 25 metros de diámetro tensado bajo ésta sobre el presbiterio. En él, la exaltación del tema eucarístico se mezcló con la identificación de ingravidez y espiritualidad; derivando en un programa ideológico-místico, justificación de la espectacular solución estructural conseguida.

El altar fue proyectado por Soteras junto a los arquitectos Vilaseca y Riudor. La estructura, calificada de contemporánea y audaz por M anuel de Solà M orales i Roselló7, se basó en un sistema tensado contrapesado, buscando dar el máximo voladizo posible frente a los espectadores de la Avenida (Fig. 1).

El proyecto se podría dividir en dos partes. A pie de suelo se proyectó una estructura en grada, bajo la cual se al bergó un mínimo programa de servicio. Unos apoyos simples de ladrillo permitieron sustentar una serie de tres plataformas escalonadas de madera de configuración geométrica variable. El nivel inferior, Iongitudinal frente a la avenida, de planta rectangular, dispuso a lado y lado del eje principal espacio para el obispado. El nivel intermedio, de planta pentagonal, permitía ubicar bajo palio a los cardenales en torno al altar. El presbiterio se situó en un nivel superior, de geometría también pentagonal, extendida mediante dos alas en sus lateral es para albergar "bajo palio" al nuncio papal y las autoridades pertinentes.

Las diferentes plataformas de madera se remataban en un voladizo perimetral, dotando a la estructura del conjunto de una cierta apariencia de ligereza. Simultáneamente, daban lugar a un ascenso escenográfico sobre el eje del altar, culminado por la presencia de una gran cruz de 35 metros de altura sobre las plataformas de madera. El ancho de tablón utilizado, equivalente al canto del escalón, junto a la variación geométrica en planta, reforzaba la materialización de los diversos estratos ascendentes (Fig. 2).

La segunda parte del proyecto, y literalmente colgando sobre la anterior, es una gran cubierta circular, plana en su cara inferior y lentiforme en la superior, de un espesor variable entre los $30 \mathrm{~cm}$ de su perímetro y un máximo de $80 \mathrm{~cm}$ en la zona central. La cubierta se proyectó inclinada sobre el eje longitudinal del altar, con mayor altura en el frente, incrementando el efecto ascensional de las plataformas.

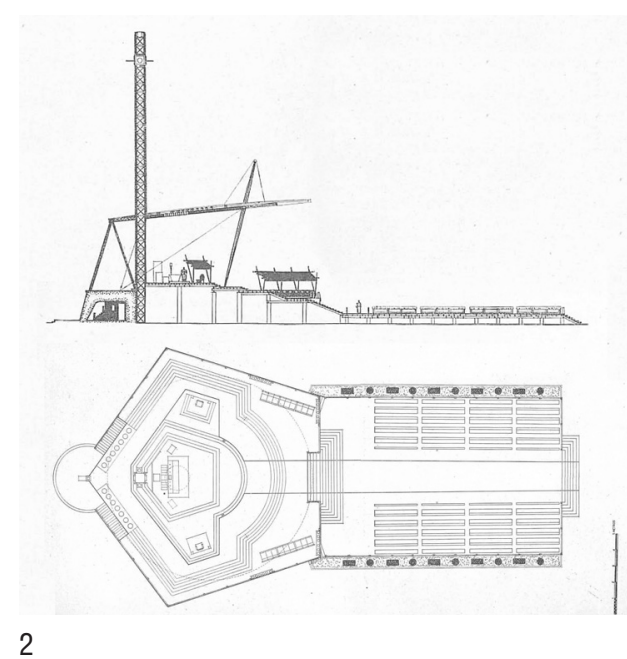

Fig. 1. Soteras, Vilaseca y Riudor. Altar Mayor del XXXV Congreso Eucarístico Internacional. Vista frontal. Cuadernos de Arquitectura, 1953, 16.

Fig. 2. Soteras, Vilaseca y Riudor. Altar Mayor del XXXV Congreso Eucarístico Internacional. Planta y sección. Montaje del autor sobre Cuadernos de Arquitectura, 1953, 16.

7. DE SOLÁ MORALES Y ROSELLÓ, Manuel, "Altar de la plaza de Pío XII para los actos del XXXV Congreso Eucarístico Internacional de Barcelona", en Cuadernos de Arquitectura, 1953, 16, pp. 41-44. 


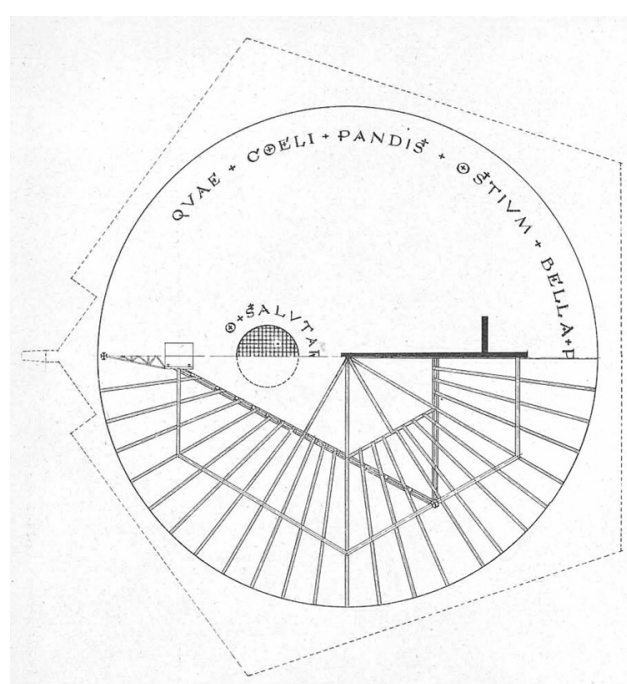

Fig. 3. Soteras, Vilaseca y Riudor. Altar Mayor del XXXV Congreso Eucarístico Internacional. Planta detalle de la estructura de la marquesina. Montaje del autor sobre Cuadernos de Arquitectura, 1953, 16.

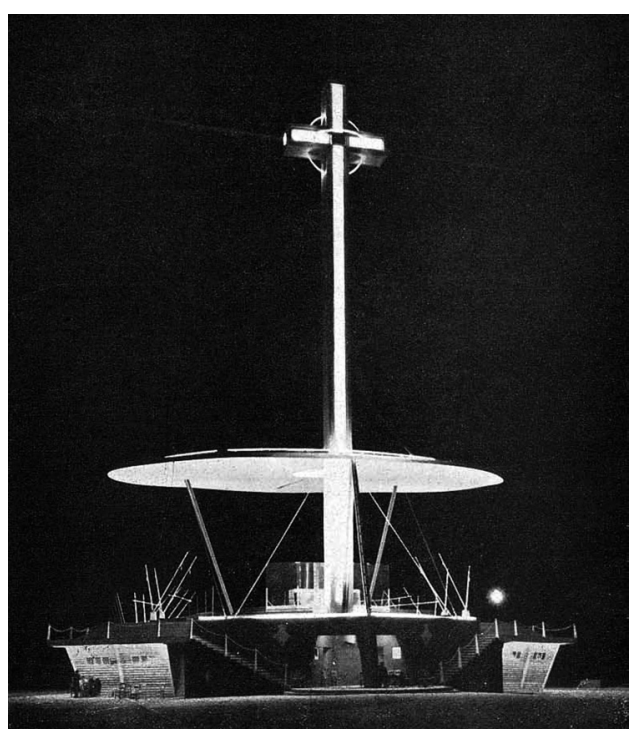

Fig. 4. Soteras, Vilaseca y Riudor. Altar Mayor del XXXV Congreso Eucarístico Internacional. Vista frontal. Cuadernos de Arquitectura, 1953, 16.

8. "El arquitecto don José Soteras Mauri dio en el Fomento de las Artes Decorativas una conferencia sobre el tema «E Palacio municipal de Deportes», refiriéndose a la celebración de los II Juegos Mediterráneos el próximo mes de julio, la necesidad de que Barcelona cuente con un edificio cubierto apto para la celebración de deportes y detalló las previsiones que se han adoptado para dicha finalidad. Hizo un elogio del proyecto premiado en el concurso celebrado el año 1951, debido a los arquitectos Barroso, Aburto y Marcide, y que no pudo llevarse a la práctica por su coste elevadísimo, por la premura de plazo disponible y por la gran extensión de terreno que requería". La Vanguardia Española, 23 de marzo de 1955, p. 16
Tres elementos de apoyo (representando según Solá M orales las tres virtudes: fe, esperanza y caridad) sostenían la estructura de cubierta. Bien fuesen la fe, la esperanza o la caridad, dos de ellos se materializaron en dos puntales metálicos atravesando la propia cubierta. Estos se realizaron en sección cruciforme, compuesta mediante cuatro perfiles en T soldados mediante tramos de cordón por su alma, de inevitable reminiscencia miésica.

El tercer apoyo aparente se realizó sobre la propia estructura de la cruz. Tras ella, e inadvertido desde el eje, se dispuso un elemento de estructura metálica en celosía formando una $\checkmark$ invertida. Este elemento se vinculó a un contrapeso de obra de fábrica en el nivel inferior para compensar el vuelo de la visera superior.

Los tres apoyos anteriores se dispusieron sobre un triángulo equilátero con uno de sus vértices sobre la cruz. Este triángulo da lugar a la estructura principal arriostrada de la cubierta, realizada en perfil de acero, complementada mediante una estructura secundaria en hexágono, también en perfil laminado, sobre la anterior.

Los vértices de dicho hexágono a lado y lado del triángulo base recibían sendos tensores superiores e inferiores, a fin de compensar tanto el peso propio de la cubierta como una posible succión de la visera en vuelo.

Sobre la estructura principal de acero se dispuso una secundaria, radial y de madera; de sección variable, trabada mediante una serie de anillos de acero. El conjunto se acabó en placa de yeso en su nivel inferior y se cubrió mediante una lámina doble de cartón embreado sobre latas de madera (Fig. 3).

Sobre el altar, desplazado del centro de la circunferencia superior, una circunferencia de madera en celosía cubierta mediante plástico permitía el paso de la luz natural o artificial en función del momento de la jornada.

Esta misma ligereza se buscó también en la cruz posterior, realizada mediante una estructura metálica en cel osía recubierta mediante lamas de madera. Haciendo de la necesidad virtud, al reducir el esfuerzo a viento del conjunto y dar lugar a una espectacular imagen nocturna al iluminarse con luz blanca desde el interior. Un efecto complementario al de la iluminación también blanca mediante focos inferiores del gran plano de cubierta circular, complementada por el uso de iluminación verde bajo las plataformas de madera inferiores (Fig. 4).

\section{ARCOS}

A penas tres años después de los actos del Congreso Eucarístico Internacional se organizaron en Barcelona los J uegos del M editerráneo. R eforzando las hipótesis que vinculan su crecimiento urbano con los grandes eventos realizados en ella, estos juegos iniciaron un proceso de recuperación de los equipamientos deportivos de la ciudad.

A sí, con motivo de los J uegos se organizó en 1951 un concurso para sustituir el Palacio de las A rtes Decorativas, situado en M ontjuich, por un Palacio M unicipal de Deportes adecuado para al bergar el evento deportivo. El acta del concurso dejó desierto el primer premio, proponiéndose para su construcción el segundo premio, otorgado al proyecto realizado por A burto, Barroso y M arcide. U n proyecto neoclásico de corte academicista donde destacaría la masividad y la articulación de sus tambores laterales ${ }^{8}$.

Tras años de incertidumbre, y con la premura forzada por la fecha de inauguración de los J uegos, el proyecto final mente ejecutado fue realizado por Soteras junto a Lorenzo GarcíaBarbón Fernández de Henestrosa. Éste, nacido en 1915, cursó estudios en los años de la autarquía, y se incorporó como técnico municipal a través de la Oficina Técnica de la Comisión Especial de U rbanismo. Previamente se ha descrito a Soteras como una figura incómoda para la crítica postfranquista. Difícilmente no se podría decir lo mismo de García-Barbón tras la polémica protagonizada en Arquitecturas Bis en $1974^{9}$. 


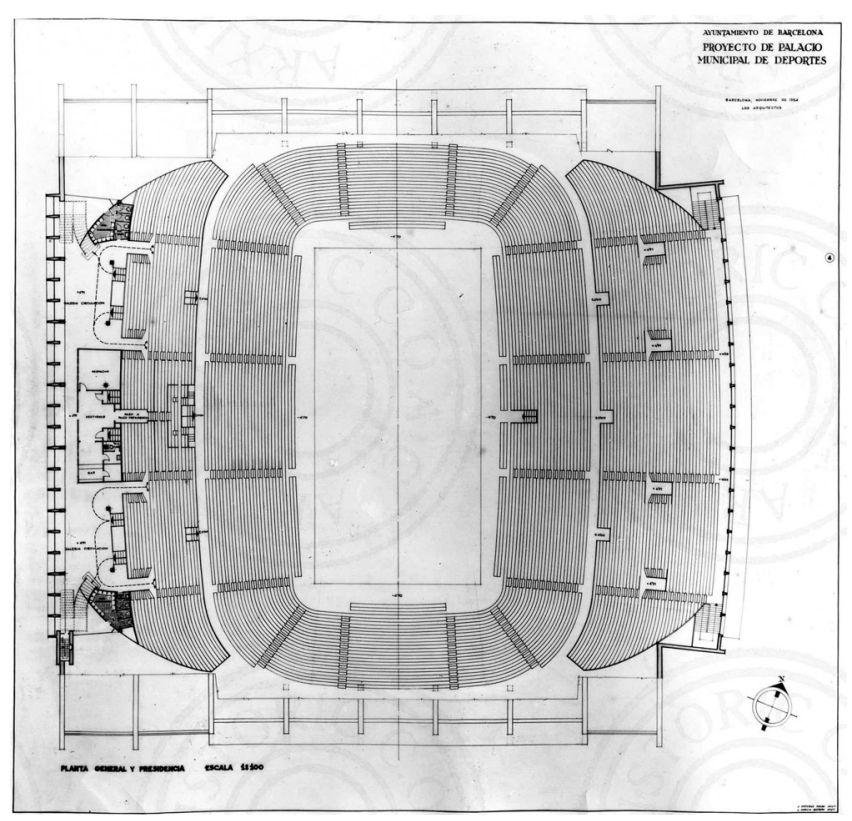

5

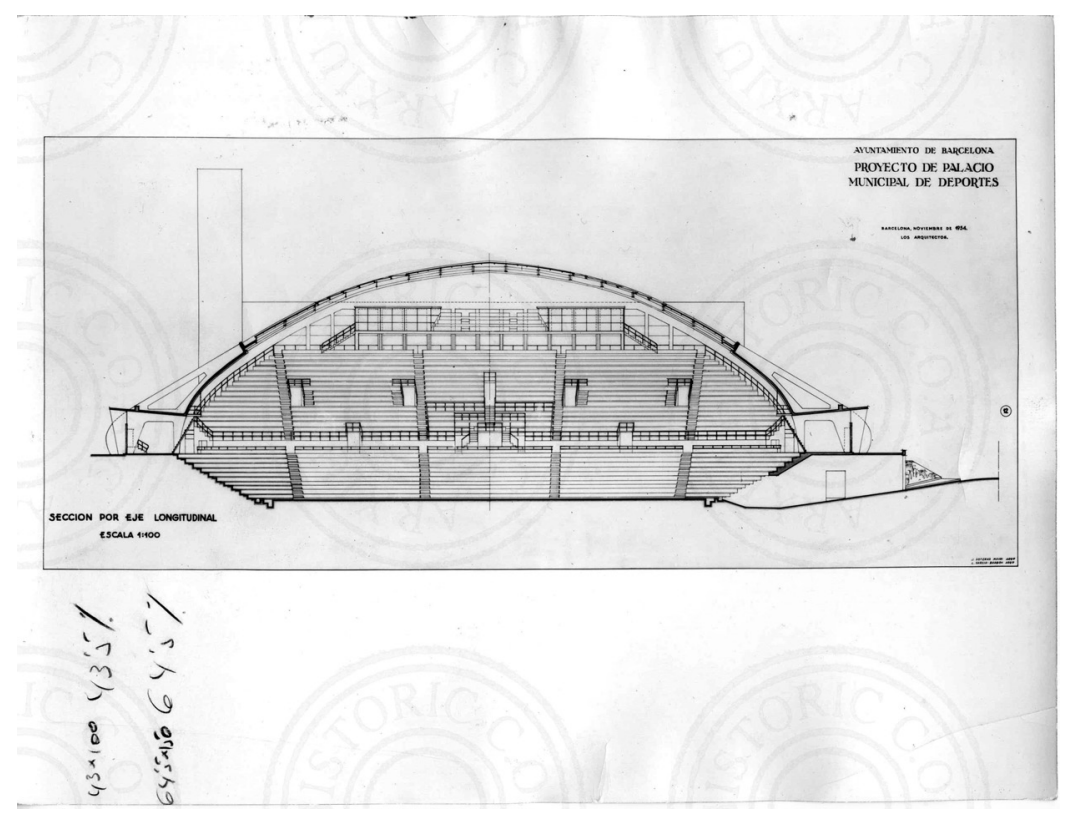

6
A mbos eran técnicos municipales y con experiencia en proyectos deportivos. Soteras había realizado con anterioridad las nuevas graderías del Estadio de Sarriá del Club Español de Barcelona. Por su parte, García-Barbón había sido el autor en 1953 de un proyecto de Estadio para el Club de Futbol B arcelona, precursor del proyecto final mente realizado entre 1954 y 1957 conjuntamente con Francisco M itjans.

La construcción del nuevo equipamiento deportivo se inició en J unio de 1954 y concluyó en Julio de 1955. En el breve periodo de un año se construyó un edificio destinado a albergar 9.500 espectadores en deporte de pista y un máximo de 12.000 en boxeo y demostraciones políticas.

La distribución de espectadores se realizó mediante una primera gradería continua en taza, construida en buena parte directamente sobre el terreno y dos graderías laterales de sección creciente en media luna. Todas el las con una cierta curvatura en planta para facilitar la correcta visión lateral de los espectadores (Fig. 5).

El edificio se caracteriza por una nave principal con nueve arcos parabólicos prefabricados de hormigón triarticulados divididos en dos partes cubriendo en el sentido de la luz mayor de la pista. La luz entre estribos del arco al canza los 65 metros, con una al tura de clave de 23,5 metros y una distancia entre articulaciones de 50,50 metros. La nave principal se reparte en 9 arcos separados 9 metros. Cada uno de los tramos de arco, de 15 toneladas de peso y con perfil en I se prefabricó en el suelo de la futura pista y se montó a posteriori, en una operación inédita en la B arcel ona de la época. La estructura principal se arriostró mediante correas también de hormigón armado en celosía. Sobre ellas se dispuso una cubierta de fibrocemento, revestida inferiormente mediante aislamiento térmico y acústico y un enlistonado enrasado al nivel superior del ala inferior de la sección de arco. Permitiendo, por tanto, una lectura espacial del trazado geométrico de las líneas de carga de la cubierta (Fig. 6).

La incidencia espacial de la estructura se revela en los dos lucernarios que recorren longitudinalmente la cubrición. A mbos se cubrieron con un sistema simple de placa ondulada de fibra de vidrio transparente "durisol", enrasado a la cara superior de cubierta, dando continuidad a la envolvente impermeable del conjunto. AI no revestirse inferiormente, estos elementos permiten hacer alarde del sistema constructivo prefabricado y ligero de la estructura (Fig 7).

La entrega inferior de la cubierta sobre los estribos se realizó mediante una cáscara nervada de hormigón armado, adaptada a la lógica de construcción in situ de todo el nivel inferior. El canto de esta cáscara arriostra lateralmente los arcos triarticulados, trabajando como una bóveda nervada. Este conjunto funciona independientemente de la estructura de los
Figs. 5 y 6. Soteras, García-Barbón. Planta General y Presidencia y sección por eje longitudinal del Palacio Municipal de Deportes de Barcelona. Archivo Lorenzo García-Barbón, COAC. Fechado noviembre 1954

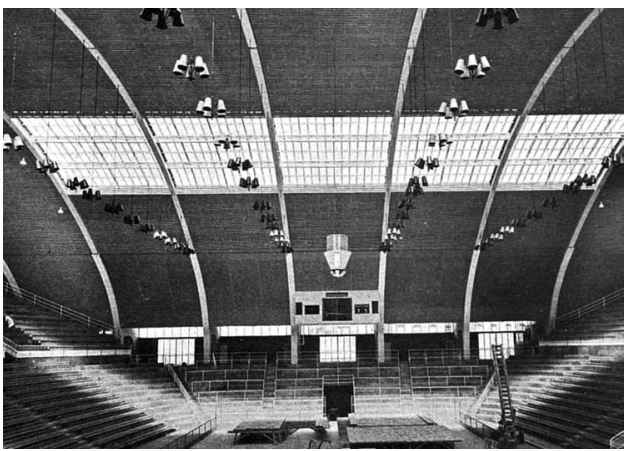

Fig. 7. Soteras, García-Barbón. Vista desde gol. Cuadernos de Arquitectura, 1955, 23
9. "Por las informaciones de prensa parece que el proyecto de esta importantísima obra se va a encargar a los arquitectos Soteras y García-Barbón. Esta elección, tan dentro de una tradición irresponsable de nuestro club en estos temas, nos parece inaceptable por dos razones fundamentales. Primera y menos importante: esta elección es ilegal (...) Segunda y más importante: estos arquitectos son malos.(...)"ANONIMO (un barcelonista arquitecto), "¿Por qué Cruyff sí y Alvar Aalto no? en Arquitecturas Bis, 1974, 1, p. 16. Véase también: REGÁS, Rosa, "El arquitecto Don Lorenzo GarcíaBarbón y Fernández de Henestrosa se querella contra Arquitecturas Bis", en Arquitecturas Bis, 1974, 3, p. 32 
Figs. 8 y 9. Soteras, García-Barbón. Sección por eje transversal y montaje con vistas del Palacio Municipal de Deportes de Barcelona. Archivo Lorenzo García-Barbón, COAC. Fechado noviembre 1954
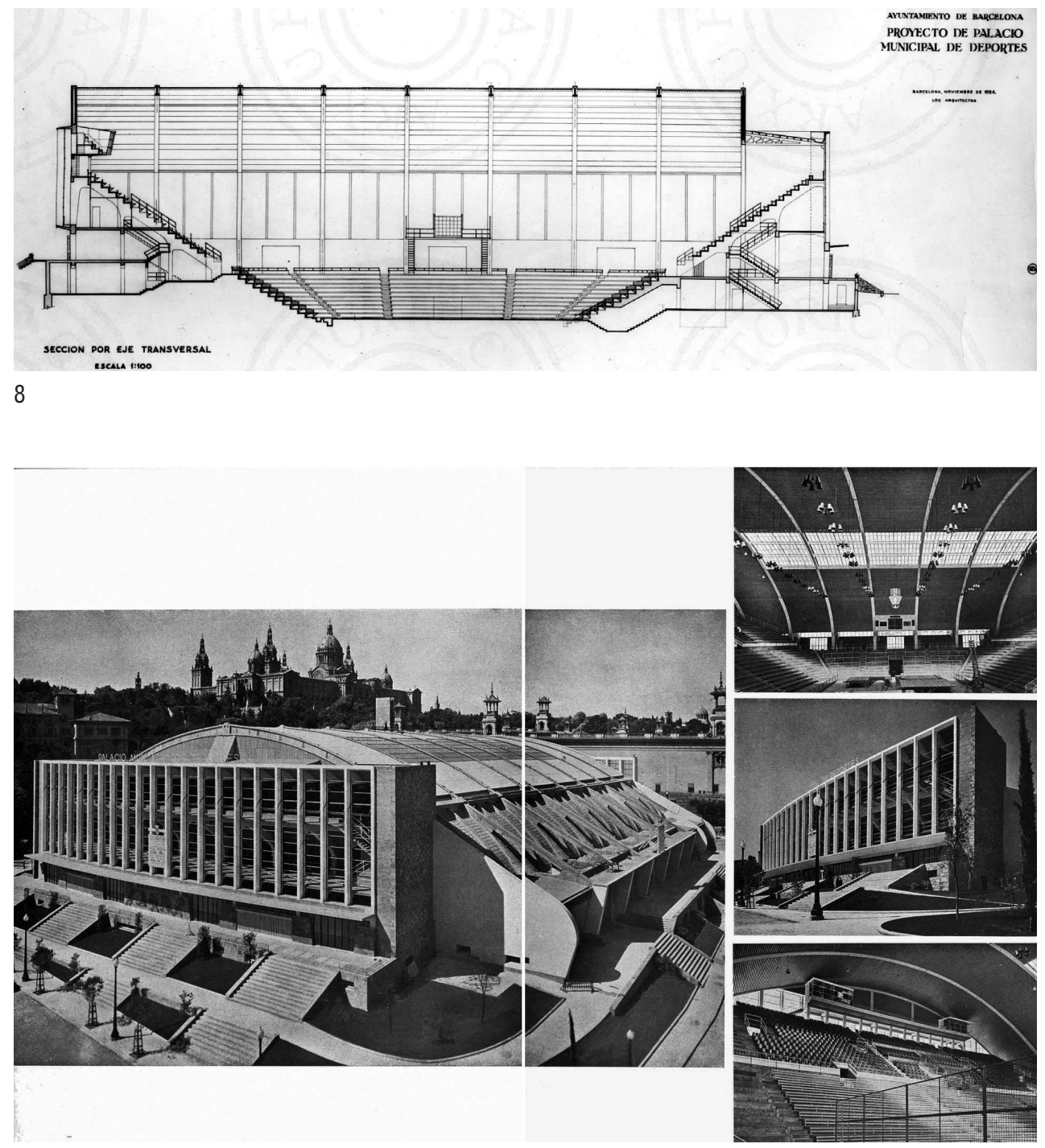

9

el ementos inferiores, tanto de las graderías en taza, encofradas directamente sobre el suelo, como de la estructura de pilares y jácenas de las graderías laterales.

Dichas graderías son simétricas en planta y asimétricas en sección, al responder a la necesidad de organizar un acceso independiente para la tribuna de autoridades y periodistas. En una muestra clara del uso mixto del edificio destinado no tan solo a actividades deportivas, sino a espacio cubierto donde realizar demostraciones y reuniones políticas.

Esta independencia de los diversos elementos estructurales se reconoce en fachada a modo de volumen dispuesto "a bofetón", frente a la directriz de la bóveda principal. El cuerpo principal curvo del edificio queda en segundo plano, al superponer frente a él un edificio-fachada frontal. Este elemento intermediador se compuso mediante estrategias propias de la modernidad, en una cel osía a modo de brise-soleil vidriada con grandes montantes vertical es revestidos en piedra artificial. B ajo este cuerpo enmarcado se organizó el acceso al edificio, resolviendo los desnivel es de la calle mediante el ementos de urbanización y rampas independientes del volumen principal (Fig. 8).

José Soteras repetiría a posteriori esta misma estrategia en la I glesia de San Pio X en el nuevo Barrio del Congreso Eucarístico, realizada entre 1958 y 1960, caracterizada por su bóveda nervada. U na estructura que inevitablemente remite a la obra de Pier L uigi N ervi10 En lo que atañe al proceso evolutivo analizado, la sección de este templo supone un paso atrás en la definición de una estructura formal a través de la estática. Al disponer dos lu- 


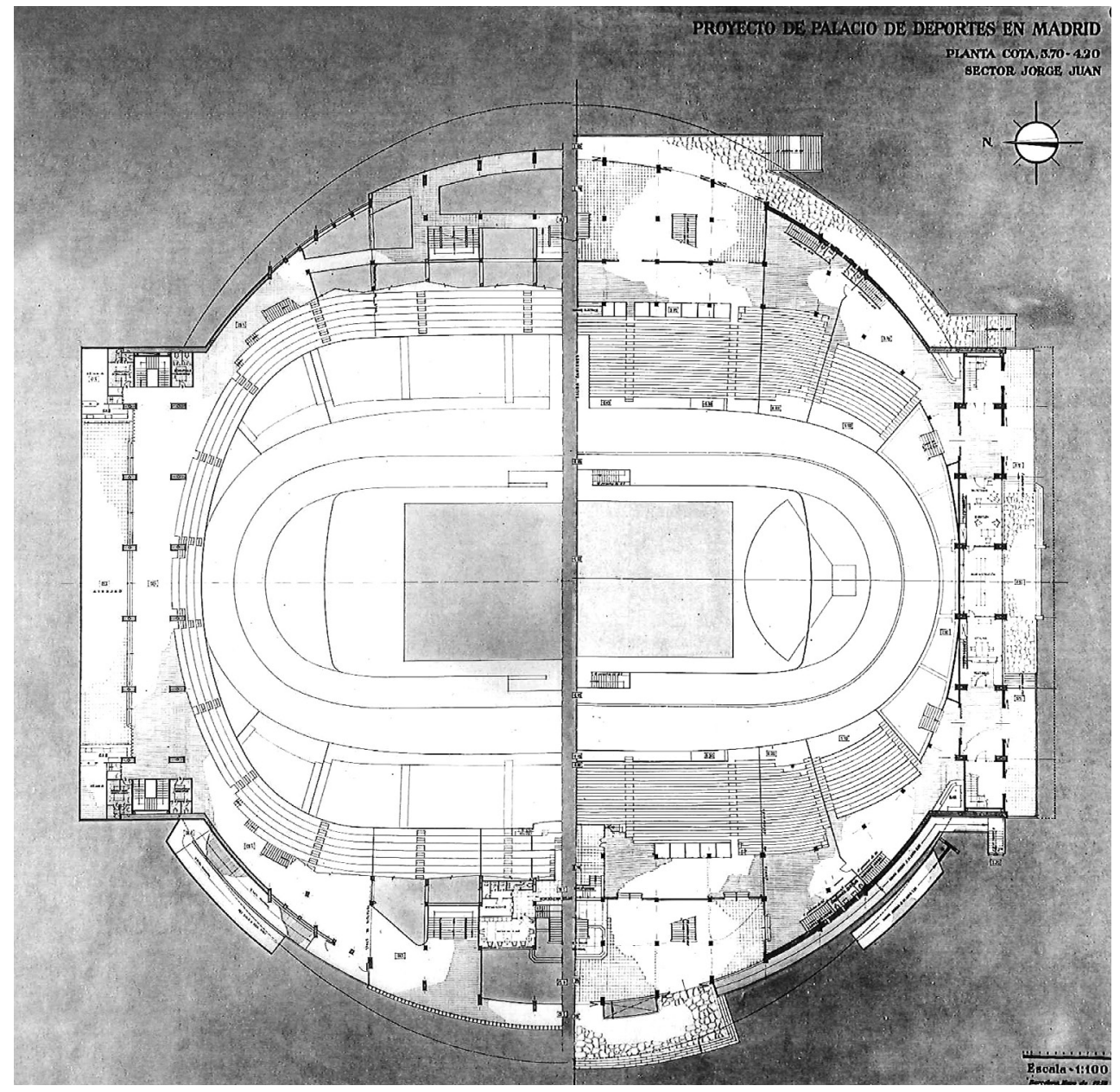

cernarios laterales, interrumpiendo la sección de bóveda, el conjunto remite a la estructura clásica de nave central y dos naves laterales propias del tipo basilical. La renuncia a la vinculación estricta entre forma, espacio y estructura para remitir a un tipo clásico, dota al conjunto de una ambigüedad inédita en los proyectos compartidos con García-B arbón.

Sorprendentemente, el nuevo Palacio M unicipal de D eportes suscitó la aprobación generalizada, recibiendo elogios públicos de personajes tan aparentemente dispares como Oriol B ohigas y del conde del A lcázar de Toledo, y Delegado Nacional de Deportes, el General M oscardón ${ }^{11}$. Coincidentes ambos en reseñar los valores de la calificada por Bohigas como "sensacional aparición de una nueva arquitectura oficial" (Fig. 9).

\section{CONTRAPESOS Y ARCOS}

Basta analizar el listado de la obra de Soteras ${ }^{12}$ para comprobar la intensidad de su trabajo en el decenio 1950-1960. En 1955, en pleno proceso de definición del Proyecto Ejecutivo del Estadio para el Club de Futbol Barcelona, Soteras y García-B arbón presentaron una propuesta al concurso del Palacio de D eportes de M adrid. Un anteproyecto, a la postre, ganador del concurso convocado por la Delegación N acional de Deportes, todavía presidida en aquel momento por el General Moscardó, quien traspasaría apenas meses después.

El proyecto supone una evolución del esquema aplicado en el proyecto de Barcelona, condicionado por la ampliación del programa de necesidades. El número de espectadores pasa a ser de 12.000 en deportes de cancha, y de 16.000 en demostraciones políticas y deportes como el boxeo. El proyecto incluía también un velódromo en su programa, además de una pista de hockey de 30x60 metros. Multiplicándose por tanto la superficie del proyecto, que en $B$ arcel ona era de 78,5x81,70; pasando a una planta general de geometría curva inserta en un cuadrado de $126 \times 136$ metros (Fig. 10).
Fig. 10. Soteras, García-Barbón. Montaje del autor con plantas del doble nivel de accesos del Palacio Municipal de Deportes de Madrid. Archivo Lorenzo García-Barbón, COAC. Fechado marzo 1956
11. Véase. GARCÍA, Santiago, "El teniente general Moscardó pasó revista al Palacio Municipal de Deportes a punto de ser inaugurado", La Vanguardia Española, 2 de Julio de 1955, p. 23. También BOHIGAS, Oriol, "El Palacio Municipal de Deportes”, Destino, 1955, 937, p. 16

12. Disponible y catalogado parcialmente en el Archivo Histórico del COAC, tras cesión de sus familiares. No así el Archivo de Lorenzo García-Barbón, también cedido al COAC pero aún hoy pendiente de catalogación. 
Fig. 11. Soteras, García-Barbón. Vista interior del Palacio Municipal de Deportes de Madrid. Archivo Lorenzo GarcíaBarbón, COAC.

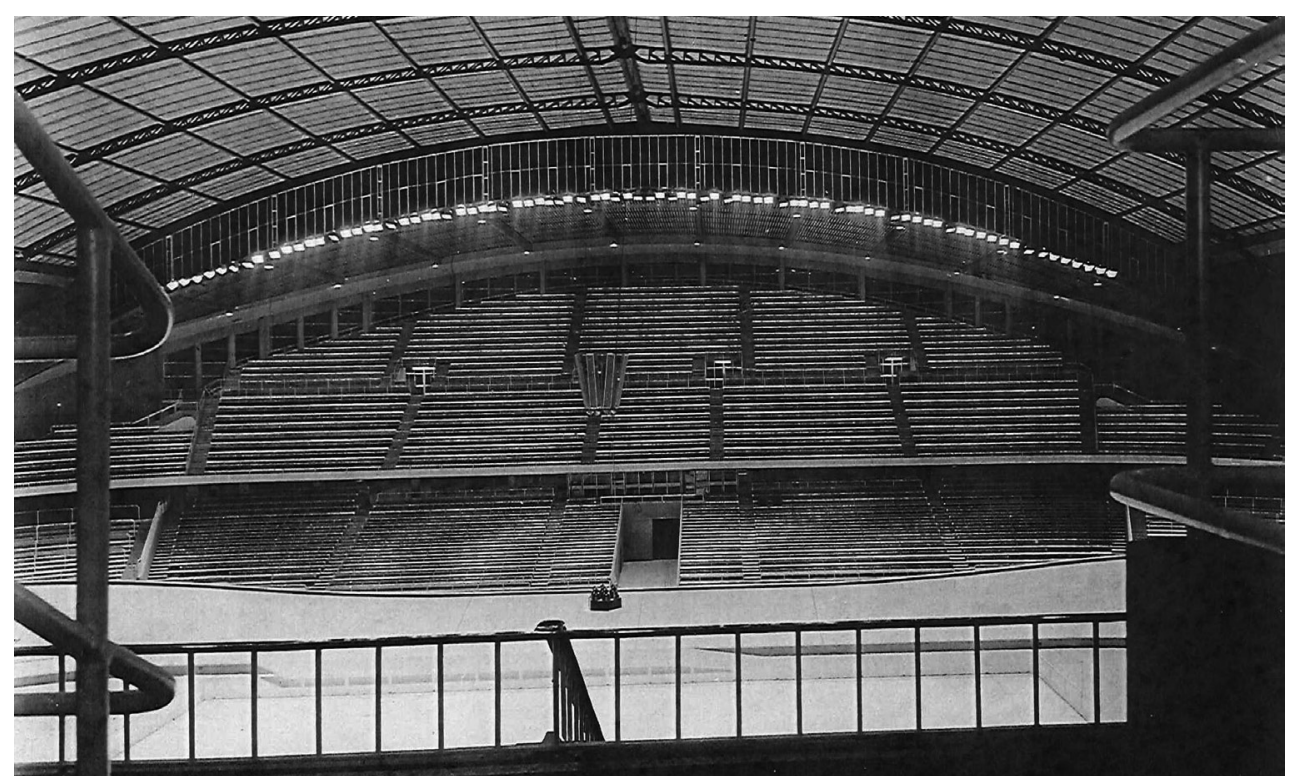

L a estructura se real izó de nuevo mediante un sistema de arcos parabólicos triarticulados prefabricados cubriendo la luz mayor de la pista, entregados sobre unos estribos de hormigón in situ. Los arcos, de 73 metros entre apoyos y 30 metros de al tura en su clave, cubrían una distancia total de 96,70 metros. En este caso toda la estructura mencionada se realizó mediante celosías metálicas vistas (Fig. 11).

A la manera de lo realizado en el Palacio de Deportes de B arcelona, los arcos se apoyaban en un sistema de estribos realizados en hormigón armado encofrado in situ. L a sección de este elemento se sofistica, al incluir una gran ménsula en voladizo que combina la lógica estructural con la espacial. U n el emento hábil para remarcar los accesos laterales al edificio mientras compensa los empujes laterales del arco. Estos se arriostran mediante una lámina de hormigón nervada, dando continuidad a la geometría del arco. Como ya sucediera en Barcelona, la coincidencia de plano de ambos nervados permite percibir la transmisión de cargas a la base de la estructura.

La principal diferencia en este caso estribaría en la sección perpendicular al eje de los arcos. U na vez más, los graderíos mayores se dispusieron adecuadamente en el sentido longitudinal del terreno de juego. La cubrición de los arcos mayores coincide prácticamente con el límite de la estructura de la primera gradería. El espacio de los lateral es de la segunda gradería, cubierto en el Palacio de Deportes de Barcelona mediante la continuidad de la bóveda, se cierra superiormente mediante una estructura de pilar con ménsula superior y viga colgada del arco principal.

A sí, a la catenaria de los arcos extremos de cubrición de la pista se le añadió la sobrecarga de una serie de tensores colgando la estructura principal de cubierta de las graderías lateral es. Este plano de cuel gue se aprovechó además para la disposición de unos lucernarios laterales, con la consiguiente tensión en una sección donde el punto de apoyo es el punto de mayor entrada de luz al espacio interior (Fig. 12).

En consecuencia, el cierre que en Barcelona era un edificio en bofetón, en Madrid se transforma en una oportunidad para contrapuntar la geometría curva de la cubierta con la geometría también curva de la envolvente de la gradería superior. Dando lugar a una forma arquitectónica derivada directamente de la combinación de geometría y estructura, sin imposiciones estilísticas (Fig. 13).

L a simultaneidad entre el proyecto del Estadio para el Club de Futbol B arcel ona (1954-57) y el Palacio de Deportes de M adrid podría explicar una cierta continuidad de soluciones. No en vano, la marquesina de tribuna del estadio se definió originalmente mediante un sistema de vigas metálicas contrapesadas, colgadas mediante un sistema de tensor y pendolón (Fig. 14). 


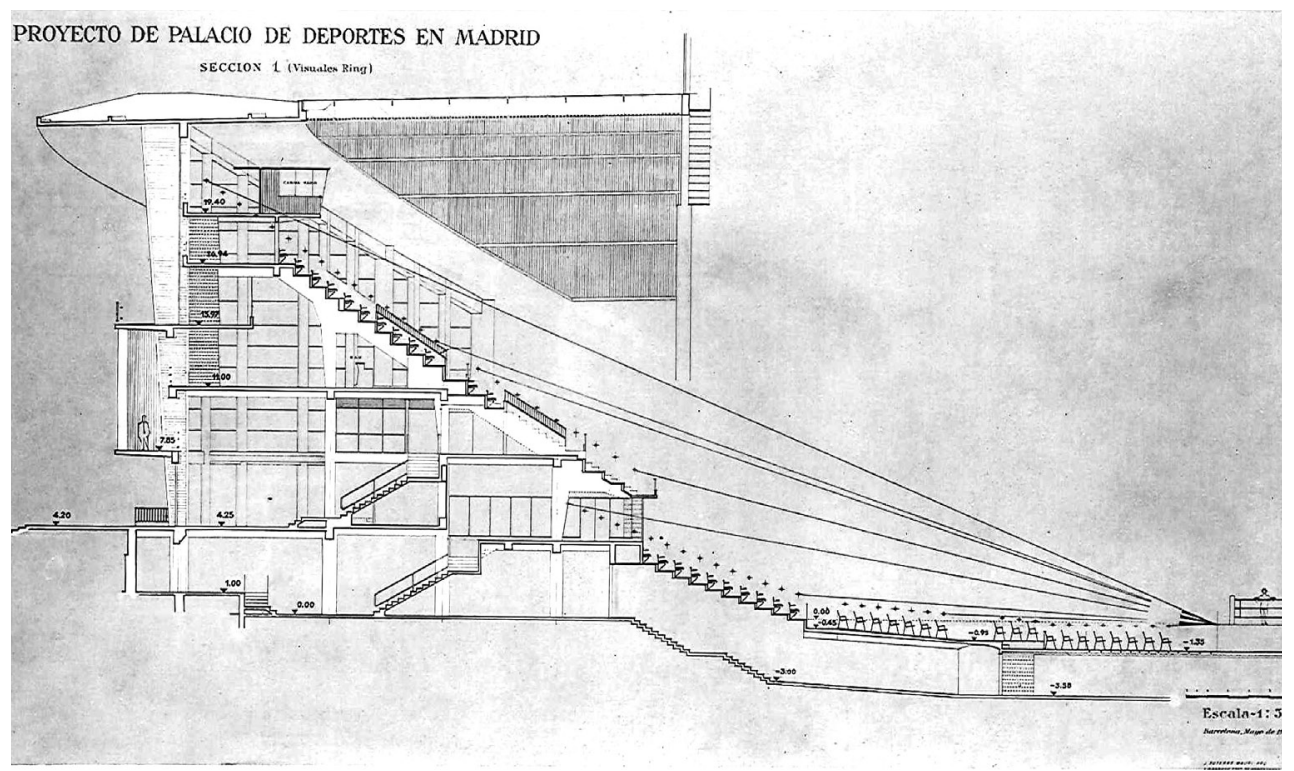

Figs. 12 y 13. Soteras, García-Barbón. Sección detalle de estructura de tribunas de gol y fachada a la Calle Goya del Palacio Municipal de Deportes de Madrid. Archivo Lorenzo García-Barbón, COAC. Fechado marzo 1956.

Fig. 14. Mitjans, Soteras, García-Barbón. Sección de la tribuna de autoridades del Estadio del Club de Fútbol BarceIona. Fechado 1954. Archivo del Centro de Documentación Barcelonista.

12

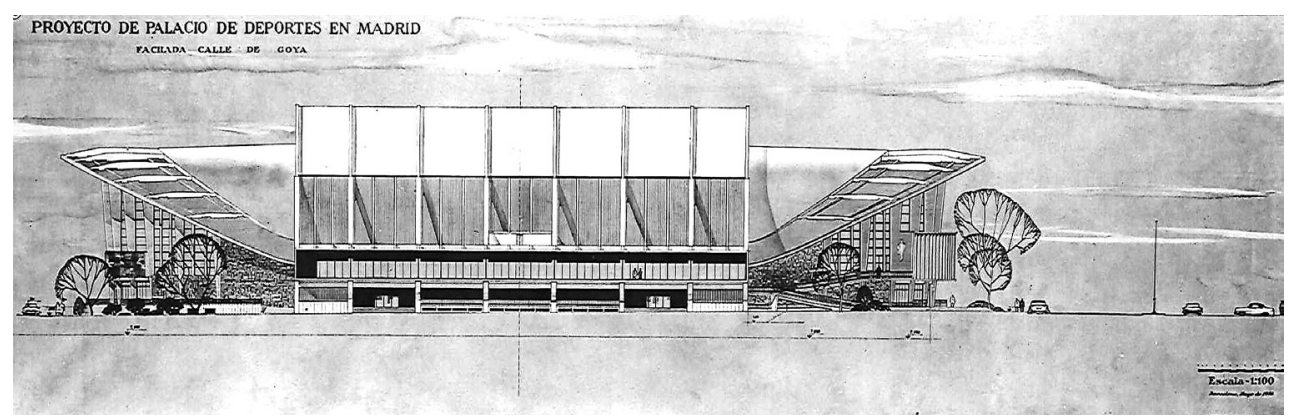

13

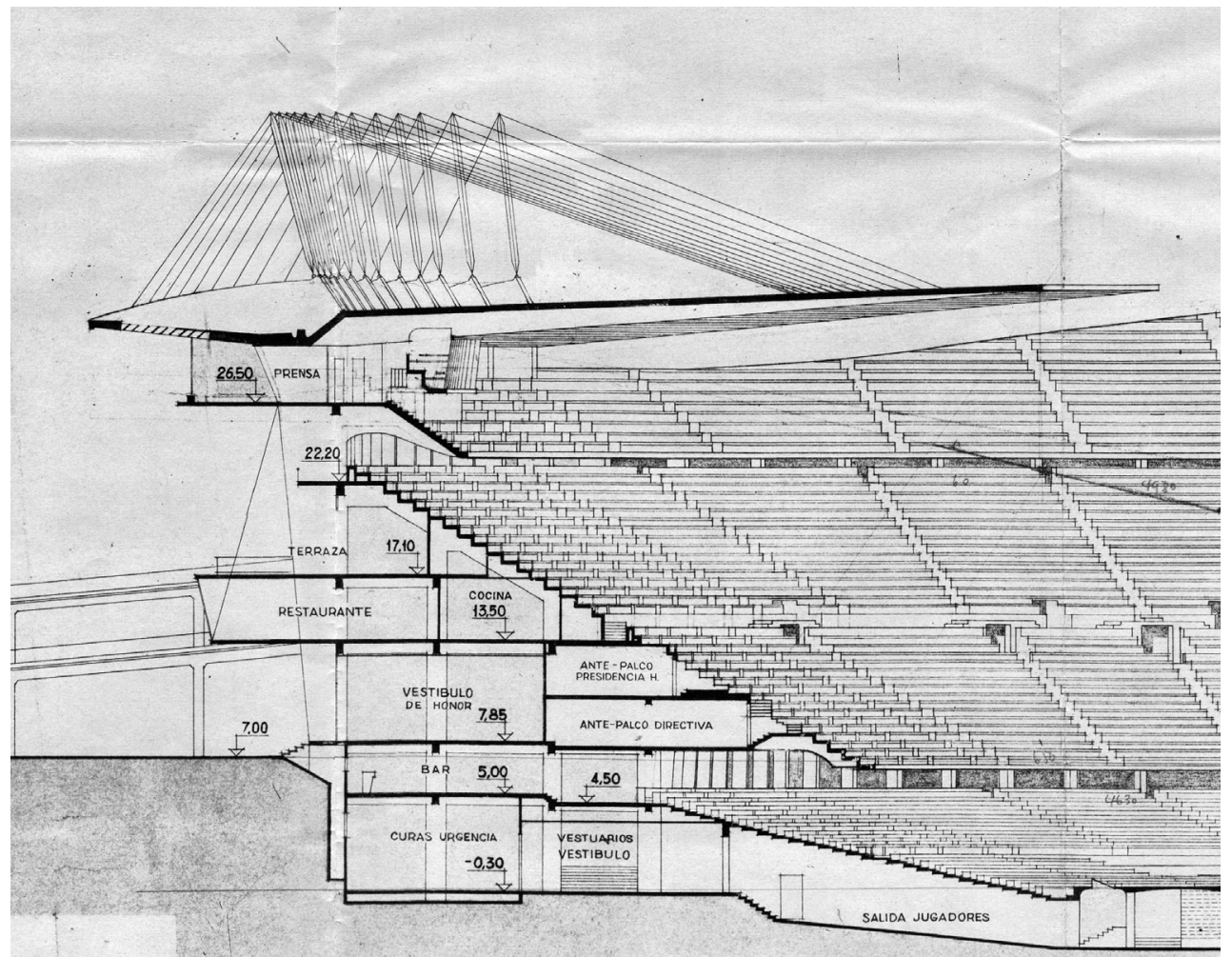


13. Véase GRAU, Ferran. Proyectos del realismo crítico en la era de la simultaneidad. Debates sobre la gestión de la información y las actuaciones en la ciudad construida. Mateo Martínez, Josep Lluís; Bru Bistuer, Eduard (dir), tesis docto ral inédita, Departamento de Proyectos Arquitectónicos UPC Barcelona, 2013, p. 304
El uso como orden mayor de fachada del sistema de pilar y ménsula, también ensayado en el Estadio del Club de Fútbol Barcelona, se equilibra visualmente mediante la aparición de elementos secundarios en el cierre, adaptando la escala del conjunto. L a estructura, protagonista de la fachada, cambia de posición y se escamotea en el plano superior, dando lugar a una lectura de la geometría de la cubierta más propia de una lámina curvada. Y final mente se esconde en el tramo intermedio entre tribuna y arco en los grosores de aislamiento, cediendo el valor espacial en el interior a la estructura de arcos metálicos.

Del mismo modo, en el exterior, es el sistema de pilar ménsula y contrapeso dota al conjunto de una expresión formal no impostada.

\section{CONCLUSIONES}

Todos los casos anteriores comparten una característica propia de una práctica donde la precariedad de medios dictaba la necesidad de soluciones austeras. No es otra que el trabajo de la estructura por forma, reduciendo a mínimos las cantidades de material necesarias para la construcción del conjunto; al trabajar sobre la lógica geométrica de la estática.

Esta práctica difícilmente podría ser calificada directamente de realista, por abandonar en cierto modo la búsqueda de soluciones desde sistemas convencionales ${ }^{13}$. Pero sin duda afronta desde un propositivo pragmatismo la resolución de problemas, en la búsqueda de un cierto límite tecnológico de lo estandarizado.

El análisis de los tres casos precedentes permitiría trazar una serie de líneas de continuidad en la definición espacial de las diferentes obras. Donde la definición del espacio en este fragmento de la obra de Soteras junto a García-B arbón se vincula a la legibilidad de las cuestiones estructural es y constructivas del proyecto, por encima de la posible estructura formal propia del tipo, aún sin abandonar ésta. Situándose en el límite superior de vinculación entre forma y estructura el Palacio de Deportes de M adrid, una obra hoy total mente desfigurada.
Igracio López Alonso. Doctor arquitecto desde 2014 con la tesis "Francesc Mitjans y el Camp Nou. Estudios previos, Proyecto Ejecutivo y dirección de obra 1954-1957". Profesor Asociado en el Departamento de Proyectos Arquitectónicos de la ETSAB/UPC desde 2008. Ha impartido clases entre otras, como Visiting Scholar en la Tsinghua University of Beijing en el periodo 2010-2011 y como Specialist Lecturer en la AVA University of East London entre 2008 y 2010. Su obra con Lagula Arquitectes ha sido seleccionada para los European Union Prize for Contemporary Architecture-Mies van der Rohe Award y para los premios FAD. 